Case Report

\title{
An Atypical Local Vesicular Reaction to the Yellow Fever Vaccine
}

\author{
Robert H. Wauters *, Camellia L. Hernandez and Maureen M. Petersen \\ Walter Reed National Military Medical Center, Bethesda, MD 20889, USA; \\ camellia.l.hernandez.mil@mail.mil (C.L.H.); maureen.m.petersen.mil@mail.mil (M.M.P.) \\ * Correspondence: robert.h.wauters.mil@mail.mil; Tel.: +1-301-400-2581 \\ Academic Editors: Alexander Zakhartchouk and Rachel L. Roper \\ Received: 2 August 2017; Accepted: 14 September 2017; Published: 19 September 2017
}

\begin{abstract}
Yellow fever vaccine is a live attenuated viral inoculation indicated for patients traveling to endemic areas. The vaccine is generally well tolerated with minimal adverse effects. Typical side effects include malaise, pain at the injection site, and, albeit rarely, immediate hypersensitivity reactions. We present a case of a rare adverse reaction to yellow fever vaccine in which a patient developed vesicular lesions resulting in bullae and circumferential hyperpigmentation.
\end{abstract}

Keywords: yellow fever vaccine; adverse reaction; blisters; rash; hyperpigmentation

\section{Case}

A 31 year old female with no significant medical history presented to the travel clinic for guidance prior to travel to South Africa and Zimbabwe. Her travel plans also included a stop in Ethiopia requiring documentation of having received the yellow fever vaccine. She endorsed feeling well and at baseline health at the time of her evaluation. Her medical history was only notable for chronic rhinitis treated with loratadine and intranasal fluticasone. The patient was provided appropriate travel guidance and administered $0.5 \mathrm{~mL}$ of an intramuscular Sanofi Pasteur typhoid vaccine in the left deltoid and $0.5 \mathrm{~mL}$ of a subcutaneous Sanofi Pasteur yellow fever vaccine injected inferiorly and distal to the insertion of the deltoid muscle. The procedure was well tolerated with no immediate adverse events.

The day after inoculation, the patient noted a small blister overlying the yellow fever inoculation site (Figure 1). Over the ensuing days, multiple serosanguinous vesicles developed, with many coalescing into a primary $4 \mathrm{~cm}$ bulla. The area was pruritic and erythematous with underlying induration (Figure 2). The patient denied any systemic symptoms including fever. Five days post-vaccination she presented to a local emergency room where the bullous lesions were unroofed. She was discharged home with topical antibiotic ointment and advised to keep the area clean and covered. On the following day, the area surrounding the newly denuded skin was noted to be hyperpigmented (Figure 3).

The patient denied ever receiving a yellow fever vaccine and had no history of a reaction to any vaccine in the past, or history of contact dermatitis. She was closely monitored and had complete resolution of her symptoms within 3 weeks. 


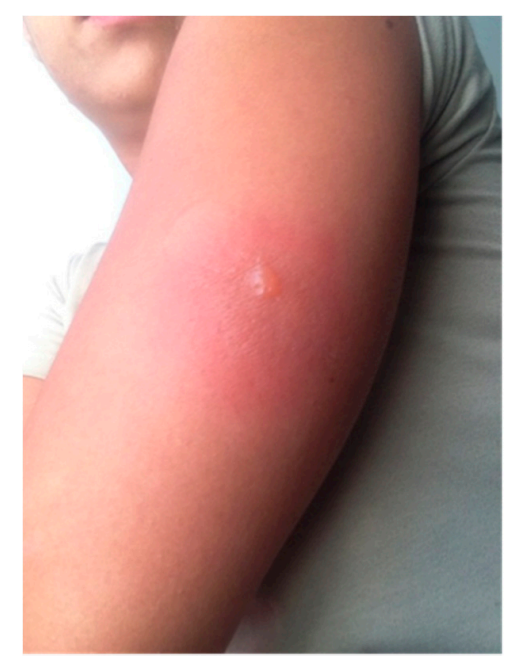

Figure 1. Day 2 following inoculation with initial formation of vesicles with surrounding erythema.

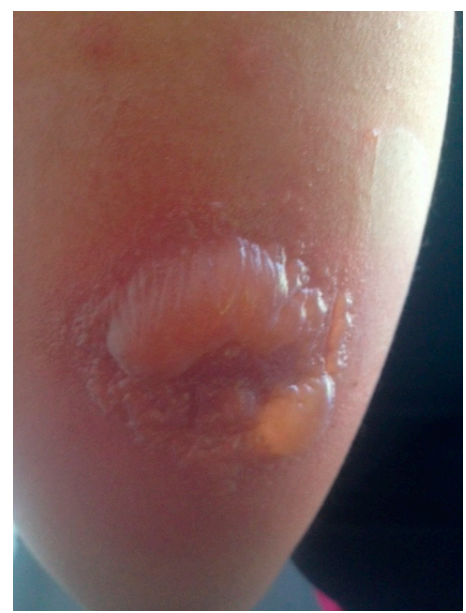

Figure 2. Day 5 after inoculation, the reaction site with multiple vesicles coalescing into a primary $4 \mathrm{~cm}$ bulla.

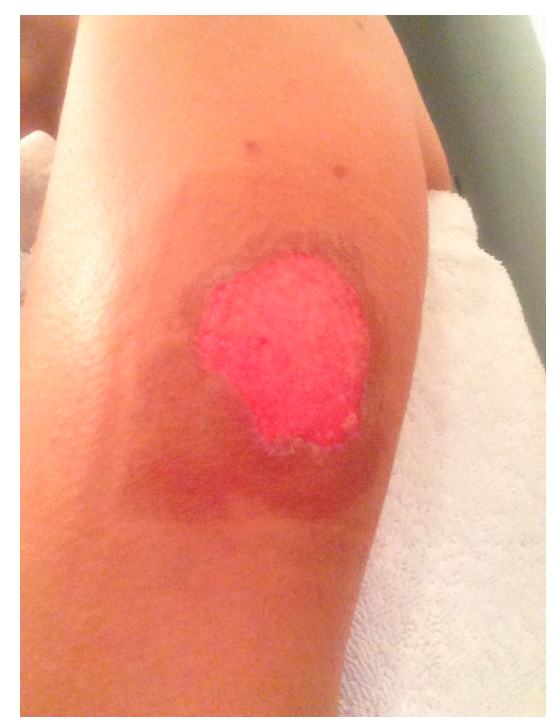

Figure 3. Day 7 post-inoculation with newly denuded skin with circumferential hyperpigmentation. 


\section{Discussion}

Live attenuated yellow fever vaccines have been available since the 1930s and are generally considered safe and effective [1]. Known adverse systemic reactions to the yellow fever vaccine include headaches, myalgia, and low-grade fevers, which typically resolve in 5-10 days. Local reactions including edema, pain, and erythema are also common [2]. Rarely, more serious neurological adverse effects have been reported in the form of meningitis and meningio-encephalitis, with rates of occurrence as high as 1:10,000 [3]. Multi-organ failure, as defined by the Brighton Collaboration criteria, has also been identified as a rare adverse effect following yellow fever vaccination [4]. Anaphylaxis, though rare, has been seen in patients with a history of an allergy to eggs or other components of the vaccine [5]. Death has been reported at a rate of 0.1-2.6 per one million administered doses of yellow fever vaccine [6]. Our patient's vesicular lesions with coalescence into bullae and hyperpigmentation is not a known or documented adverse reaction to the vaccine, and prompted further investigation using the Vaccine Adverse Event Reporting System (VAERS).

The VAERS database contains information on unverified reports of adverse events following immunization with US-licensed vaccines. VAERS was accessed using the Center for Disease Control Wide-Ranging Online Data for Epidemiologic Research (CDC WONDER) tool. The initial search of the entire database from its first year of tracking in 1990 through May 2017, yielded 30 case reports of a blistering reaction following administration of the yellow fever vaccine [7]. Meticulous review of each case identified 12 cases that noted the formation of vesicular lesions at the yellow fever inoculation site and 18 cases that reported vesicular lesions following the vaccine but occurring in areas other than the injection site.

The following discussion pertains to the 12 cases that reported vesicular formation near the inoculation site (Table 1). Case 1 described a small single vesicle occurring at the vaccine injection site. Cases 2-10 reported multiple vesicles occurring at the yellow fever inoculation site; however, no skin pigment changes developed or were reported. Cases 11 and 12 reported a reaction similar to our patient's case. Case 11 described a patient who developed a large bulla with circumferential hyperpigmentation seven days after immunization. Case 12 described a patient with erythema, induration, and a coalescing bullous lesion at the inoculation site associated with surrounding hyperpigmentation. The time course of events in this case was not reported. The patient in Case 11 took no concomitant medications, and the patient in Case 12 took only fish oil and a daily multivitamin [7].

Table 1. 12 VAERS cases of local vesicular reaction to the yellow fever vaccine.

\begin{tabular}{ccc}
\hline Case & VAERS ID & Description \\
\hline 1 & $041172-1$ & Single small blister \\
2 & $258521-1$ & Erythema, multiple vesicles, pruritus \\
3 & $261259-1$ & Vesicles, pruritus, erythema \\
4 & $306400-1$ & Erythema, multiple vesicles \\
5 & $391917-1$ & Erythema, multiple vesicles \\
6 & $419936-1$ & Erythema, multiple vesicles \\
7 & $421959-1$ & Vesicles, pruritus \\
8 & $453003-1$ & Erythema, multiple vesicles, pruritus \\
9 & $559035-1$ & Vesicles, induration, erythema \\
10 & $337338-1$ & Erythema, large vesicle \\
11 & $390002-1$ & Bulla, circumferential hyperpigmentation 7 days after vaccination \\
12 & $471071-1$ & Coalescing vesicles, hyperpigmentation \\
\hline
\end{tabular}

The possible etiologies of our patient's reaction include a type IV hypersensitivity to the vaccine or its components. The yellow fever vaccine is known to contain live attenuated virus, gelatin, and sorbitol. A review of the literature identified multiple published reports of a gelatin hypersensitivity causing symptoms of anaphylaxis, but there has never been a published case report of a type IV hypersensitivity reaction to gelatin [8]. Sorbitol has also been implicated, albeit rarely, in type I 
hypersensitivity, but there are currently no known cases of a contact dermatitis to sorbitol [9-12]. Live viral replication and activity at the inoculation site was considered as a possible etiology of our patient's symptoms; however, the bulla was unroofed and treated at an outside emergency room, and no specimen was taken for the purpose of reverse transcription polymerase chain reaction analysis. Our patient was concomitantly administered typhoid vaccination and was taking a second generation antihistamine, which we considered as potentially playing a role in her reaction. However, the two reported similar cases, Case 11 and 12, were not associated with antihistamine use or concomitant typhoid vaccination, and we think this is likely not an important contributing factor. It is unknown whether our patient became seropositive for yellow fever after her reaction. The patient is an active duty military service member and is no longer in the country. Additionally, it is not standard protocol to test for seroconversion in deploying forces.

\section{Conclusions}

Our patient's case demonstrates a rare and significant adverse reaction to the yellow fever vaccine. Yellow fever is found in tropical and subtropical areas in South America and Africa, and travel to these areas warrants administration of the vaccine [13]. Early identification of vaccine adverse reactions, including rare and previously not described events, is important to health care providers in immunology, infectious disease, and primary care to ensure appropriate counseling of possible adverse events as well as prompt and appropriate treatment of these maladies.

Author Contributions: R.H.W., C.LH., and M.M.P. participated in the drafting and critical revision of the manuscript. All authors approved the final version.

Conflicts of Interest: The authors have no conflicts of interest to declare pertaining to this article. The views expressed in this article are those of the authors and do not reflect the official policy of the Department of the Army, Navy, Department of Defense, or U.S. Government. Address correspondence to Robert Wauters, MD, Allergy, Immunology, and Immunization Service, 8901 Wisconsin Avenue, Bethesda, MD 20889-5600, USA; e-mail address: robert.h.wauters.mil@mail.mil.

\section{References}

1. Lindsey, N.P.; Rabe, I.B.; Miller, E.R.; Fischer, M.; Staples, J.E. Adverse event reports following yellow fever vaccination, 2007-13. J. Travel Med. 2016, 23. [CrossRef] [PubMed]

2. Yellow Fever Vaccine YF-VAX. (June 2016). Package Insert. Available online: https://www.fda.gov/ downloads/biologicsbloodvaccines/vaccines/approvedproducts/ucm142831.pdf (accessed on 15 July 2017).

3. Guimard, T.T. Short report: Incidence of yellow fever vaccine-associated neurotropic disease. Am. J. Trop. Med. Hyg. 2009, 81, 1141-1143. [CrossRef] [PubMed]

4. Thomas, R.E. Yellow fever vaccine-associated viscerotropic disease: Current perspectives. Drug Des. Dev. Ther. 2016, 10, 3345. [CrossRef] [PubMed]

5. Sampson, H.A. Food allergy: A practice parameter update. J. Allergy Clin. Immunol. 2014, 134, $1016-1025$. [CrossRef] [PubMed]

6. Amanna, I.J.; Slifka, M.K. Questions regarding the safety and duration of immunity following live yellow fever vaccination. Expert Rev. Vaccines 2016, 15, 1519-1533. [CrossRef] [PubMed]

7. Centers for Disease Control and Prevention. Vaccine Adverse Event Reporting System (VAERS). 2017. Available online: https: / / wonder.cdc.gov/vaers.html (accessed on 15 July 2017).

8. Sakaguchi, M.; Nakayama, T.; Inouye, S. Food allergy to gelatin in children with systemic immediate-type reactions, including anaphylaxis, to vaccines. J. Allergy Clin. Immunol. 1996, 98 Pt 1, 1058-1061. [CrossRef]

9. Hino, H.; Kasai, S.; Hattori, N.; Kenjo, K. A case of allergic urticaria caused by erythritol. J. Dermatol. 2000, 27, 163-165. [CrossRef] [PubMed]

10. Shirao, K.; Inoue, M.; Tokuda, R.; Nagao, M.; Yamaguchi, M.; Okahata, H.; Fujisawa, T. "Bitter sweet": A child case of erythritol-induced anaphylaxis. Allergol. Int. 2013, 62, 269-271. [CrossRef] [PubMed]

11. Sugiura, S.; Kondo, Y.; Ito, K.; Hashiguchi, A.; Takeuchi, M.; Koyama, N. A case of anaphylaxis to erythritol diagnosed by CD203c expression-based basophil activation test. Ann. Allergy Asthma Immunol. 2013, 111, 222-223. [CrossRef] [PubMed] 
12. Yunginger, J.W.; Jones, R.T.; Kita, H.; Saito, K.; Hefle, S.L.; Taylor, S.L. Allergic reactions after ingestion of erythritol-containing foods and beverages. J. Allergy Clin. Immunol. 2001, 108, 650. [CrossRef] [PubMed]

13. Center for Disease Control and Prevention. Yellow Fever. Available online: https://www.cdc.gov/ yellowfever/index.html (accessed on 15 July 2017).

(c) 2017 by the authors. Licensee MDPI, Basel, Switzerland. This article is an open access article distributed under the terms and conditions of the Creative Commons Attribution (CC BY) license (http://creativecommons.org/licenses/by/4.0/). 